\title{
Are interstellar ices porous, and how do the pores collapse?
}

\author{
C. R. Hill ${ }^{1}$, C. Mitterdorfer2, T. G. A. Youngs ${ }^{3}$, D. T. Bowron ${ }^{3}$,

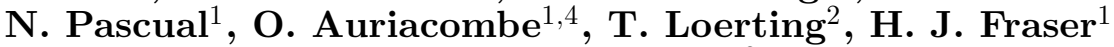 \\ and Susanne Höfner ${ }^{2}$ \\ ${ }^{1}$ The Open University, UK ${ }^{2}$ University of Innsbruck, ${ }^{3}$ STFC, ISIS, ${ }^{4}$ RAL Space \\ email: catherine.hill@open.ac.uk
}

\section{Introduction}

Amorphous solid water (ASW) is of great importance in astrochemistry as it has been detected in star forming regions, comets, and cold solar-system objects. A key property of ASW is its porous nature (with the extent of porosity reflecting the formation and growth conditions) and the subsequent pore collapse when the ice is heated. If interstellar ices are porous there are huge implications to both the process of planet formation and the budgets of molecular gas in the solid and gas phases. It is therefore vital to understand ASW porosity over astronomically relevant conditions in order to effectively model its potential effects on these processes.

Small angle neutron scattering is a non-destructive technique, which is suitable for studying porous structures with dimensions between 10 and 1000, giving information on pore size, shape and surface area (in contast to other experimental techniques previously used to investigate ASW pore collapse which only give information on the fact and not the nature of collapse). Here we present new results from two experiments designed to unravel the mechanisms underpinning pore formation, evolution and collapse in ASW. First, a sample of compact ASW (c-ASW), is warmed from 80 to $144 \mathrm{~K}$ to study the pore collapse kinetics. Our results show that cASW is porous, although these pores are likely to be closed to the vacuumice interface. However, unlike previous models, our data shows pore collapse has a sudden onset at $121 \mathrm{~K}$, with no evidence for pore-clustering. Furthermore we refute the claim that pore collapse can proceed as either a function of time or temperature as our isothermal studies at 92 and $117 \mathrm{~K}$ show that the pores do not change appreciably over timescales of hours. Using our second experiments, we show how this technique can be exploited to study ice growth in situ, and illustrate how porosity varies as a function of growth conditions and temperature. We conclude by placing these results back in context, discussing how our data modify the modelling constraints on the porosity of interstellar water ice from pre-stellar through to proto-stellar regions. 